\title{
LOS TRABAJOS PARA CASA: PERSPECTIVAS DE PADRES, ALUMNOS Y PROFESORES. UN ESTUDIO DE CASO.
}

\author{
TRABALHOS PARA CASA: PERSPECTIVAS DE PAIS, ALUNOS E PROFESSORES. \\ UM ESTUDO DE CASO
}

\section{HOMEWORK: PARENTS, STUDENTS AND TEACHERS’ PERCEPCIONS. A CASE STUDY}

\author{
Esther Nieto Moreno de Diezmas* \\ Maria da Conceição Figueira Santos Pereira*
}

\begin{abstract}
Resumen: En este trabajo se analizan prácticas relativas a los trabajos para casa (TPC) en los tres momentos de su desarrollo: (i) prescripción por el profesor en el aula, (ii) realización del alumno en casa y (iii) verificación y corrección en el aula. Los TPC se desenvuelven en dos contextos distintos: escuela y entorno familiar e implican a tres actores distintos: profesores, alumnos y padres, por lo que el objetivo principal de este estudio es comprender mejor las opiniones de cada uno de estos colectivos respecto a esta práctica pedagógica. Se han obtenido datos a partir de la realización de entrevistas y tres cuestionarios adaptados de Antunes (2012), Pires (2012) y Simão et al. (2012) dirigidos a padres $(n=62)$, alumnos $(n=81)$ y profesores $(n=4)$ de tres niveles educativos: $2^{\circ}, 3^{\circ}$ y $4^{\circ}$ año del primer ciclo de educación primaria (ensino básico). El análisis de los datos indica la existencia de prácticas tradicionales en la prescripción de los TPC que impiden que se aprovechen al máximo las potencialidades para el aprendizaje de esta herramienta educativa. En la última parte del trabajo se identifican las áreas de mejora detectadas en el estudio y se incluyen una serie de recomendaciones referidas a aspectos como frecuencia, autorregulación, tipo de tareas, participación parental, corrección y evaluación.
\end{abstract}

Palabras clave: Trabajos para casa. Percepciones de padres y profesores. Aprendizaje. Educación primaria.

\begin{abstract}
In this paper, practices related to homework in its three stages of development are analysed: (i) assigment by the teacher in the classroom, (ii) realization at home by the student, and (iii) verification and correction in the classroom. Homework is carried out in two different contexts: school and home, and involves three different actors: teachers, students and parents, and therefore, the main objective of this study is to better understand the views of each of these groups regarding this pedagogical practice. Data have been collected from interviews and three questionnaires adapted from Antunes (2012), Pires (2012) and Simão et al. (2012) aimed at parents $(n=62)$, students $(n=81)$ and teachers $(n=4)$ from three educational levels: 2nd, 3rd and 4th of primary education (ensino básico). The analysis of the data indicates the existence of traditional practices in the assignment of homework that frustrate its potential to contribute to learning. In the final part of the paper, areas for improvement are identified, and a series of recommendations are included regarding issues such as frequency, self-regulation, type of work, parental participation, correction and evaluation.
\end{abstract}

Keywords: Homework assignments. Primary education. Perceptions of families. Parents and teachers.

\footnotetext{
*University de Castilla-La Mancha. E-mail: esther.nieto@uclm.es

** Facudade de Psicologia e Ciências da Educação - Lisboa, Portugal. E-mail: clanca@eselx.ipl.pt
} 
Resumo: Neste trabalho analisam-se práticas relativas aos trabalhos para casa (TPC) nos três momentos do seu desenvolvimento. i) prescrição pelo professor na aula, ii) realização pelo aluno em casa, iii) verificação e correção na aula. Os TPC desenvolvem-se em dois contextos distintos: escola e envolvimento familiar e implicam três atores distintos: professores, alunos e pais, pelo que o objetivo principal deste estudo é compreender as opiniões de cada um destes protagonistas a respeito desta prática pedagógica. Para o efeito recolheram-se dados a partir de entrevistas e de três questionários adaptados de Antunes (2012), Pires (2012) e Simão et al. (2012) destinados a país, (n=62), alunos $(n=81)$ e professores $(n=4)$ do $2 .^{\circ}, 3 .^{\circ}$ e $4 .^{\circ}$ ano do $1 .^{\circ}$ ciclo do ensino básico. A análise dos dados indica a existência de práticas tradicionais na prescrição dos TPC que impedem que se aproveite ao máximo as potencialidades desta ferramenta educativa. Na última parte do estudo identificam-se aspetos relevantes desta prática e incluem-se algumas recomendações relacionadas com a frequência na prescrição dos TPC, autorregulação, tipo de tarefas, participação parental, correção e avaliação.

Palavras chave: Trabalhos para casa. Aprendizagem. Perceção de pais e professores. Ensino básico

\section{Introducción}

Los trabajos para casa (TPC) se definen como aquellas tareas que los profesores mandan a los alumnos para que las hagan fuera del horario escolar (COOPER, 1989) y su objetivo fundamental es promover oportunidades complementarias de aprendizaje fuera de la escuela.

Por definición, constituyen un puente o instrumento de conexión entre la escuela y las familias, ya que a través de las tareas los padres pueden tener conocimiento de los contenidos que se están trabajando en el aula, cómo se están trabajando y cuáles son las dificultades de sus hijos. De este modo, por medio de los TPC los actores sociales implicados "se conocen virtualmente" (HENRIQUES, 2006, p. 225).

Sin embargo, la pluralidad de actores que se conjugan en esta práctica pedagógica puede ocasionar fricciones y suscitar actitudes negativas por parte de los alumnos que los definen como "trabalhos para carecas, trabalho para cábulas, trabalho para camelos, tortura para crianças, trabalho p'ra chatear...” (ARAÚJO, 2009, p. 30), y por parte de los padres, que los consideran estresantes (BALLI, 1998) y motivo de conflictos familiares (DUDLEY-MARLING, 2003;
KRAVOLEC; BUELL, 2000). Además, los TPC pueden causar desigualdades entre los alumnos por el nivel sociocultural de los padres (CARVALHO; SERPA, 2006)

No obstante, y a pesar de que la investigación se encuentra también dividida entre defensores y detractores, los (TPC), constituyen una práctica pedagógica asumida como común en la escuela. (HONG; WAN; PENG, 2011; SIMÕES, 2006; ZABALZA, 2001). Sin embargo, la ausencia de regulación normativa de esta práctica, así como el escaso número de estudios realizados en el contexto portugués, dejan abiertos multitud de interrogantes acerca de su metodología, impacto y opinión de los diferentes agentes involucrados: alumnos, profesores y familias. En este trabajo, pretendemos comprender las diferentes perspectivas de profesores, padres y alumnos y las diversas prácticas desarrolladas en los procesos de prescripción, realización y corrección de los TPC en el contexto de $2^{\circ}$, $3^{\circ}$ y $4^{\circ}$ de educación primaria.

\section{Revisión de la literatura de investigación sobre los TPC}

Dado el esfuerzo y la inversión temporal que alumnos, familias y profesores realizan 
en el desarrollo de la práctica pedagógica de los TPC, una de las líneas principales de investigación estudia su utilidad y repercusión en los aprendizajes. Cooper y sus colaboradores han dedicado un buen número de estudios (COOPER, 1989, 2001; COOPER; LINDSAY; NYE; GREATHOUSE, 1998; COOPER; ROBINSON; PATALL, 2006; COOPER; VALENTINE, 2001) a verificar el impacto de los TPC en el rendimiento escolar. Sin embargo, las relaciones entre estas dos variables han resultado ser más débiles de lo esperado, y en todo caso, los resultados académicos de los alumnos de primaria se ven mucho menos beneficiados por la realización de las tareas en casa que los de los estudiantes de secundaria. Hay autores como Marzano y Pickering (2007) que muestran un análisis más optimista del impacto de las tareas en la mejora del rendimiento escolar, pero también encontramos investigadores como Kohn (2006) que, tras revisar estudios anteriores, concluye que los datos indican que hasta el momento, no ha podido demostrarse que la realización de las tareas de casa contribuyan a una mejora significativa de los resultados académicos. Incluso Cooper y colaboradores, grandes defensores de los TPC, han reconocido que "no strong evidence was found for an association between the homework-achivement link and the outcome mesure” (COOPER et al., 2006, p. 1).

$\mathrm{Al}$ no poderse demostrar que los TPC puedan repercutir de una manera significativa en el rendimiento, la investigación se ha dirigido a detectar otro tipo de beneficios de los TPC conectados con aspectos metacognitivos y emocionales. Los TPC se revelan, según varios estudios, como una práctica que fomenta el aprendizaje autorregulado (BEMPECHART, 2004; BEMBENUTTY, 2009; TRAUTWEIN; KOLLER, 2003) y contribuye al desarrollo de hábitos de estudio, autodisciplina, responsabilidad y gestión del tiempo (KITSANTAS; ZIMMERMAN, 2009). La motivación, autorregulación y la implicación en la tarea del alumno parecen factores clave para que las TPC sean eficaces.

Otros estudios encuentran conexiones entre TPC y rendimiento analizando variables como la instrumentalidad, dificultad y satisfacción percibidas (ROSÁRIO; MOURÃO; BALDAQUE; NUÑEZ; GONZÁLEZ-PIENDA; CEREZO; VALLE, 2009).

Otra de las líneas de investigación de los TPC se centra en la implicación parental. Aunque existen estudios (LEVIN; LEVYSHIFFF; APPRLBAUM-PELED; KATZ; KOMAR; MEIRAN, 1997) que concluyen que la ayuda de los padres en los TPC no influye en el rendimiento, e incluso pueda perjudicarlo (COOPER; LINDAY; NYE, 2000; EPSTEIN, 1988) debido a la existencia de técnicas de implicación parental nocivas, son más las voces que defienden que los aspectos positivos de la participación parental superan a los negativos y que hay que identificar las conductas adecuadas de apoyo a las TPC para formar a profesores y padres en prácticas efectivas de acompañamiento (ALLEMAN; BROPHY; KNIGHTON; LEY; BOTWINSKI; MIDDLESTEAD, 2010; WALKER; HOOVER-DEMPSEY; WHETSEL; GREEN, 2004).

También se han estudiado diversos aspectos prácticas de la realización de los TPC en cada una de sus fases. Según Cooper (2001) el TPC se entiende como un proceso temporal tripartito que integra las fases de preparación o prescripción en el aula, la realización en casa y, por último, la corrección o feedback por parte del profesor. En este contexto, los elementos que más atención han suscitado han sido el tiempo de realización de los TPC, el tipo de tarea y el proceso de corrección. 
A pesar de que los alumnos emplean parte de su tiempo libre en realizar sus TPC, el tiempo no es una variable que afecte al rendimiento (ROSÁRIO; MOURÃO; NUÑEZ; GONZÁLEZ-PIENDA; VALLE, 2006), ya que, como es lógico, los alumnos con mayor rendimiento resuelven antes sus tareas que los menos aventajados (PAN; REGUEIRO; PONTE; RODRÍGUEZ; PIÑEIRO; VALLE, 2013). La atención de los investigadores se ha dirigido a determinar el tiempo que los alumnos pasan realizándolos y establecer límites. Cooper (1989) estudia la relación entre la cantidad de tiempo invertida en la realización de los TPC en el contexto de educación secundaria y concluye que a partir de las 2 horas diarias se disminuye su efecto positivo e incluso puede llegar a ser contraproducente. Para el contexto de educación primaria se establece la regla de los 10 minutos (COOPER; ROBINSON; PATTAL, 2006), de modo que el tiempo de realización de los TPC no debería exceder del resultado de multiplicar el curso en el que se encuentra el alumno por 10 minutos. Sin embargo, diversos estudios confirman la falta de observancia de estas limitaciones temporales. Henriques (2006) detecta que alumnos de primaria invierten más de una hora y muchas veces hasta 2 para hacer sus TPC y en secundaria se han hallado medias superiores a las 3 horas (GALLOWAY; CONNER; POPE, 2013, p. 490).

También se ha dirigido la atención a la tipología y caracterización de las tareas propuestas. Para Bembenutty (2011), para que sean eficaces, las tareas han de ser significativas y Alleman y otros (2010) exponen 7 principios para lograrlo, que incluyen emplear la diversidad del alumnado como fuente de aprendizaje, aplicar los aprendizajes en situaciones reales y utilizar las tareas para mantener actualizado el currículo. Diversos estudios muestran, sin embargo, la existencia de hábitos pedagógicos tradicionales de los TPC como su prescripción masiva e indiferenciada y su uso para repasar o adelantar contenidos y así ganar tiempo para poder impartir todo el programa (HENRIQUES, 2006). Además, se detectan contextos en que los TPC consisten en tareas repetitivas como copias, cálculos y fichas del libro (ARAÚJO, 2009; HENRIQUES, 2006). También la corrección adolece de prácticas poco beneficiosas para que los TPC sean eficaces y contribuyan al desarrollo de los aprendizajes, de modo que se ha detectado que los profesores no siempre corrigen los TPC (HENRIQUES, 2006; SIMÃO, 2012) y que muchas veces no existe corrección individual ni feedback adecuado.

En este trabajo, se aportarán nuevas evidencias acerca de la caracterización de la práctica pedagógica de los TPC y de las percepciones de familias, alumnos y profesores.

\section{Preguntas de investigación}

El objetivo fundamental de este trabajo es proporcionar información acerca de la práctica educativa de los trabajos para casa, y concretamente dar respuesta a las preguntas que se plantean sobre la cuestión. La principal pregunta de investigación que guía este trabajo es la siguiente:

¿Cómo se desarrollan los tres momentos clave en la práctica de los TPC, es decir la prescripción en el aula, su realización en el aula y su corrección por el profesor?

De esta cuestión principal se derivan o desglosan otras preguntas como:

¿Cuál es la utilidad que los distintos agentes perciben en esta práctica pedagógica?

¿Cuáles son las prácticas más habituales según padres, alumnos y profesores? 
¿Cuál es la opinión de los padres respecto de su necesidad?

¿Las prácticas son eficaces para la contribución de los TPC a la adquisición de competencias, destrezas y conocimientos?

\section{Metodología}

Teniendo en cuenta el objeto de estudio y sus finalidades, se emplearon técnicas de investigación mixtas.

Se han recabado datos, a través de entrevistas semiestructuradas con los profesores y por medio de cuestionarios (SOUSA; BAPTISTA, 2011). Para reforzar la fiabilidad de los resultados de investigación se ha recurrido a la triangulación de los datos (DENZIN et al., 2006) por medio de la recogida de información de los tres actores implicados en la práctica pedagógica de los TPC: padres, profesores y alumnos, de manera que el acceso a fuentes diferenciadas aumenta la validez interna del estudio.

\section{Muestra}

El estudio se realizó en un colegio ubicado en el barrio de Benfica, en el municipio de Lisboa (Portugal), que es un barrio popular integrado por más de 36.000 habitantes.

Participaron en el estudio 147 individuos: 81 alumnos de cuatro clases distintas, 62 padres y 4 profesores que eran los tutores de cada una de las clases objeto de la investigación. En la tabla 1 se observa el número de participantes de cada grupo.

Tabla 1: Número de participantes por año.

\begin{tabular}{l|c|c|c}
\multicolumn{1}{c|}{ CURSO/EDAD } & ALUMNOS & PADRES & PROFESORES \\
\hline $\begin{array}{l}2^{\circ} \text { año del primer ciclo de ensino básico (alumnos de 7-8 } \\
\text { años) }\end{array}$ & 25 & 20 & 1 \\
\hline $\begin{array}{l}3^{\circ} \text { año del primer ciclo de ensino básico (alumnos de 8-9 } \\
\text { años) }\end{array}$ & 20 & 17 & 1 \\
\hline $\begin{array}{l}4^{\circ} \mathrm{A} \text { año del primer ciclo de ensino básico (alumnos de } \\
9-10 \text { años) }\end{array}$ & 18 & 15 & 1 \\
\hline $\begin{array}{l}4^{\circ} \mathrm{B} \text { año del primer ciclo de ensino básico (alumnos de } \\
\text { 9-10 años) }\end{array}$ & 18 & 10 & 1 \\
\hline TOTAL & 81 & 62 & 4 \\
\hline
\end{tabular}

\section{Instrumentos de recogida y análisis de datos}

Para la realización de la investigación se solicitó autorización a la directora del centro educativo y a los padres o tutores de los alumnos en la que se especificaba el objetivo del estudio y se garantizaba la confidencialidad y el anonimato.
Para la recogida de datos se elaboraron tres cuestionarios, dirigidos a padres, alumnos y profesores, respectivamente, adaptados de Antunes (2012), Pires (2012) e Simão et al. (2012). Estos cuestionarios incluyen mayoritariamente preguntas de respuesta cerrada, aunque existen algunas preguntas de respuesta abierta para que padres, profesores 
y alumnos pudieran añadir perspectivas y opiniones no previstas en las cuestiones de respuesta cerrada.

Para validar los cuestionarios se pilotaron en otro colegio. Se aplicó a un grupo de diez alumnos, a un profesor y a un padre que no formaron parte de la investigación. El pilotaje sacó a la luz la existencia de cuestiones que podían dar lugar a diversas interpretaciones y se reformularon en pro de una mayor claridad y univocidad.

En cuanto a la aplicación de los cuestionarios, los alumnos y el profesor de cada curso los realizaron en el aula durante la clase. Por su parte, los cuestionarios para los padres se entregaron a los alumnos para que los llevaran a casa y los devolvieran cumplimentados. El número de padres participantes en el estudio es inferior al de los alumnos, dado que algunos de los cuestionarios que los estudiantes llevaron a casa, no fueron devueltos.

El tratamiento de los datos se realizó a través del software Statistical Package for the Social Sciences (SPSS), mediante la elaboración de una base de datos que sirvió para el posterior análisis de frecuencias de cada una de las cuestiones. Posteriormente se realizaron tests no-paramétricos como el test de Kruskal-Wallis y tests Post Hoc, para relacionar variables.

Las entrevistas realizadas, de naturaleza semiestructurada, permitieron profundizar en los datos expresados en los cuestionarios a padres, alumnos y profesores, y posibilitaron la posterior triangulación de datos.

\section{Resultados y discusión}

Para facilitar la presentación y discusión de los resultados, los dividiremos en tres epígrafes correspondientes a cada una de las fases que Cooper (2001) distingue en la práctica pedagógica de los TPC: la prescripción de los TPC en el aula, su realización en casa y su verificación o corrección subsiguiente.

\section{Prescripción de los TPC en el aula}

En lo que respecta a la prescripción de los TPC en el aula, presentaremos los datos referentes a los siguientes aspectos: finalidades y utilidad percibida de los TPC, frecuencia de su prescripción, tipo de tareas que integran los TPC y diferenciación de los TPC.

Los profesores prescriben los TPC porque consideran que contribuyen a la consolidación de los contenidos (50\%) o porque permiten desarrollar la autonomía y responsabilidad de los alumnos (50\%). Los padres están totalmente de acuerdo $(53,2 \%)$ o simplemente de acuerdo (38,7\%) con la afirmación de que los TPC contribuyen al aprendizaje de sus hijos y solo un 3,2\% está en desacuerdo, mientras que un $4,8 \%$ no está ni de acuerdo ni en desacuerdo. El alto porcentaje de padres que refiere una actitud positiva respecto de la utilidad de los TPC en la adquisición de los aprendizajes parece manifestarse también en el hecho de que, casi por unanimidad, estén de acuerdo con que sus hijos lleven tareas para casa, puesto que el $93,5 \%$ se decanta a favor de esta práctica educativa y solo el $6,5 \%$ se manifiesta en contra. Estos datos se encuentran en línea con otros estudios (KIEWRA; KAUFFMANN; HART; SCOULAR; BROWN; TYLER, 2010; PIRES, 2012) que revelan que, aunque existan quejas y debates en torno a los TPC, la mayoría de los padres se posiciona a favor de su prescripción.

Cuando se pregunta a los alumnos acerca de la frecuencia con la que el profesor prescribe tareas para casa, la mayoría afirma 
que ocurre diariamente (54,3\%) o casi a diario, es decir, 3 o 4 veces por semana (7,4\%). Un tercio declara tener tareas solo los fines de semana y el 4,9\% 1 o 2 veces por semana. Los padres corroboran estos datos, puesto que $67,8 \%$ atestiguan que sus hijos traen tareas a casa diariamente o 3 o 4 veces por semana. Por su parte, un 50\% de los profesores afirman prescribir TPC diariamente, un 25\% entre 3 y 4 veces por semana y el $25 \%$ restante entre 1 a 2 veces.

El test Post Hoc revela que existe correlación entre la frecuencia semanal de los TPC y el año de escolaridad, de manera que la mayor frecuencia se detecta en el $3^{\circ}$ curso. En las entrevistas con los profesores, la profesora de $3^{\circ}(\mathrm{P} 2)$ declara mandar tareas diariamente, debido a que el programa en este curso es bastante extenso y se hace necesario emplear esta herramienta para poder contar con un tiempo extra fuera de la escuela para hacer ejercicios, practicar y repasar y abordar así todos los contenidos. De esta manera, las tareas para casa se consideran como una especie de extensión de la jornada escolar. Esta concepción y finalidad de los TPC se ha identificado ya en otros trabajos (ANTUNES, 2012; HENRIQUES, 2006).

Los datos acerca de la frecuencia en la realización de los TPC muestran que las tareas forman parte de la cotidianidad de los alumnos. Esta es una realidad que ya había sido detectada en estudios anteriores. Para Henriques (2006, p. 222) “os TPC continuam na moda” y Simão (2012, p. 17), tras revisar las investigaciones realizadas en Potugal, concluye que "o recurso a estas tarefas como prática pedagógica é até agora uma realidade”. Además, la postura de los padres a favor de los TPC y la frecuencia de prescripción de las tareas parecen corresponder con algunos de los mitos señalados por Corno y Shu (2004), tales como que los padres quieren que sus hijos tengan TPC y que es mejor profesor quien manda tareas regularmente.

Cuando se pregunta a los alumnos cuál es el tipo de tarea que suelen llevar para casa, el 60,5\% de los alumnos responde que el trabajo más solicitado por los profesores son las cuentas. El tipo de tarea que los alumnos marcan como la segunda más frecuente son los ejercicios del libro, según el 59,2\%. Profesores y padres coinciden en que estos son los TPC más habituales, aunque estos dos colectivos invierten el orden de frecuencia respecto de los alumnos, refiriendo como primera opción los ejercicios del libro (según el 75\% de los profesores y 85,4\% de los padres) y las cuentas, como segunda (según el 25\% de los profesores y 56,4\% de los padres). Además el $80,6 \%$ de los padres no creen que sus hijos debieran hacer otro tipo de trabajos para casa y solo el 16,1\% aboga por la necesidad de introducir otro tipo de tareas y al ser interrogados por su naturaleza, mencionan principalmente, la realización de más investigaciones y también, más ejercicios con las tablas de multiplicar. La prescripción de TPC repetitivos y poco creativos, que simplemente reproducen los contenidos de los libros, parece ser una práctica instaurada referida en otros trabajos (ARAÚJO, 2009) y criticados por autores como Henriques (2006, p. 221) que considera que las tareas invaden el espacio familiar para, después de la jornada escolar, no aportar otra cosa que "fazer mais do mesmo".

En cuanto a la prescripción de TPC diferenciados, parece ser una práctica que se va adoptando poco a poco, ya que, aunque el $50 \%$ del profesorado encuestado refiere que todos alumnos realizan las mismas tareas, el $50 \%$ restante explica que, dado que existen alumnos con distintos niveles de logro, los TPC inciden en las particulares áreas de mejora de cada estudiante. Estos hallazgos contrastan con el estudio de Henriques 
(2006) que detecta una indiferenciación en la prescripción de los TPC, ya que el 100\% del profesorado encuestado en su estudio reconoce que los trabajos para casa que mandan a sus alumnos son iguales para todos. Sin embargo, el resultado de este estudio concuerda con el realizado unos años después por Antunes (2012), que registra que el 52,9\% del profesorado declara prever actividades diferenciadas en sus TPC. Es posible que en los últimos años se estén comenzando a implantar prácticas pedagógicas de los TPC dirigidas a aprovechar su potencial para atender a la diversidad y proporcionar un contexto adaptado a los distintos ritmos y estilos de aprendizaje de los alumnos. La prescripción de TPC que atiendan a la diversidad ha de ser un objetivo para el profesorado, puesto que como afirma Henriques (2006, p. 241) "Um trabalho prescrito, para quem o não sabe fazer, ou para quem ele se torne fácil de mais, é naturalmente um trabalho inútil”.

\section{Realización de los TPC}

La segunda fase en el ciclo de los TPC es su realización fuera del horario escolar. En este apartado, analizaremos los datos correspondientes a los aspectos prácticos del desenvolvimiento de los TPC en esta etapa de su desarrollo, y expondremos los datos recabados referentes a su lugar de realización, comprensión de la tarea y capacidad para realizarla, necesidad de ayuda para ello, tiempo empleado y, por último, estimación de la interferencia de los TPC en la realización de otras actividades y en la vida familiar.

A pesar de la denominación de estas tareas como "tareas para casa", y aunque la mayoría de los alumnos las llevan a cabo en el hogar $(77,8 \%)$, no todos ellos las realizan en el entorno doméstico, ya que un $9,9 \%$ los realizan en la CAF, y el resto en el colegio o en clases particulares. Según la percepción de los padres, el porcentaje de realización de los deberes en el hogar aumenta al 88,7\%.

Además, aunque la mayoría de los alumnos dice saber hacer los deberes (un $71,6 \%)$, más de una cuarta parte $(28,4 \%)$ declara que no siempre comprende lo que tiene que hacer o no sabe hacerlo. Este es un problema ya detectado por Henriques (2006, p. 283) en las entrevistas a los monitores de ATL (actividades de tiempo libre), quienes explicaban que muchas veces los niños no sabían lo que tenían que hacer e incluso, los propios monitores, al ser consultados, no eran capaces de ver qué es lo que el profesor pretendía con la tarea propuesta.

Las dificultades que los alumnos encuentran para finalizar con éxito sus TPC, se conecta con la cuestión de la necesidad de ayuda y apoyo parental. En este sentido, un alto porcentaje de los alumnos, el 69,1\% manifiesta contar con ayuda en la realización de sus TPC, mientras que el 30,9\% afirma no tener ninguna ayuda. El porcentaje de participación parental aumenta cuando son preguntadas las familias, ya que un 83,9\% declara ayudar a sus hijos en las tareas y solo el $16,1 \%$ no acostumbra a prestarles ayuda. Entre los padres que auxilian a sus hijos en las tareas, cerca de la mitad, 45,3\% reconocen que esta labor les resulta difícil, sobre todo debido a la divergencia entre los métodos de enseñanza con los que ellos aprendieron y los métodos adoptados por el profesor. Este es, precisamente, uno de los inconvenientes del apoyo parental detectado en estudios anteriores (PATALL; COOPER; ROBINSON, 2008). La necesidad de que las familias cuenten con las estrategias para poder ayudar a sus hijos constituye una demanda de los propios padres que había aflorado en investigaciones como la de Henriques (2006, p. 240). Los docentes expresan visiones encontradas en este tema, ya que, mientras que el $25 \%$ 
sostiene que los padres deben ayudar a sus hijos en la realización de los TPC, y el 50\% estima que esta ayuda ha de llevarse a cabo solo a veces, el $25 \%$ se posiciona en contra de que los padres intervengan en la resolución de las tareas. Estas distintas posturas acerca de la implicación parental pueden fundarse en resultados de investigación igualmente diversos, ya que, por un lado, algunos estudios detectan prácticas inadecuadas de acompañamiento parental en las familias (COOPER et al. 2000) que pueden incluso perjudicar el rendimiento de los hijos, mientras que, por otro existen investigaciones que vinculan el éxito escolar con la implicación de las familias (GONZÁLEZ-PIENDA; NÚÑEZ, 2005; PATTAL et al., 2008).

En cuanto al tiempo que los alumnos necesitan para terminar sus TPC, solo un 8,6\% de los alumnos dice emplear más de 1 hora, $16 \%$ entre 45 minutos y 1 hora, el 17,3\% entre 30 y 45 minutos, el 34,6\%, entre 15 y 30 minutos y el 23,5\%, menos de 15 minutos. La percepción de los padres difiere de la de sus hijos, estimando que estos emplean más tiempo en la realización de los TPC. Según el 29\% de los padres, sus hijos precisan de más de una hora; el 24,2\% calcula que el tiempo requerido oscila entre 45 minutos y 1 hora; el $24,4 \%$ estima que sus hijos dedican entre $30 \mathrm{y}$ 45 minutos, el 17,7\% entre 15 y 30 minutos y solo un 4,8\% percibe que sus hijos resuelven las tareas en menos de 15 minutos. Por tanto existen diferencias entre las perspectivas de estos dos grupos, puesto que la mayoría de los padres, un 53,2\% estiman que sus hijos dedican entre 45 minutos y más de una hora a los deberes, y entre ellos, un gran porcentaje, el $29 \%$ considera que precisan de más de una hora, mientras que la mayoría de los alumnos, el 58,1\% calculan que terminan sus tareas en menos de 30 minutos, y entre ellos, un buen porcentaje (el 23,5\%) estima que emplean menos de 15 minutos. En el extremo opuesto a los padres, se encuentran los profesores, ya que el $75 \%$ estima que los alumnos necesitan entre 15 y 30 minutos para terminar sus TPC y el $25 \%$, considera que emplean entre $30 \mathrm{y}$ 45 minutos. Por consiguiente, los profesores tienden a estimar que los alumnos necesitan menos tiempo para realizar los TPC que los padres y los propios alumnos, mientras que los padres consideran que el tiempo empleado por sus hijos es mayor que el percibido por los niños. Otros estudios se hacen eco de esta discordancia en la percepción del tiempo empleado entre los diferentes actores. Concretamente, el trabajo de Henriques (2006) revela que los profesores subestiman el tiempo que los alumnos precisan en la resolución de los TPC, en comparación con la opinión de los monitores de ATL que están presentes mientras los niños hacen las tareas. Mientras que para los profesores una media hora es suficiente para terminar los TPC, los ATL declaran que los alumnos emplean más de una hora y que, en ocasiones, tardan entre una hora y media y hasta dos horas y que a veces ni siquiera consiguen terminarlos en ese tiempo. Estas cifras son bastante elevadas en comparación con los resultados registrados en nuestro estudio, que se encuentra más cerca de cumplir la regla de los 10 minutos (COOPER et al. 2006), es decir, la limitación del tiempo dedicado a los deberes a los minutos resultantes de multiplicar 10 por el curso en el que se encuentra el alumno (10 minutos en $1^{\circ}$ de primaria o ensino básico, 20 en $2^{\circ}, 30$ en $3^{\circ}, 40$ en $4^{\circ}$, etc.)

En cuanto al tiempo que los TPC restan al desarrollo de otras actividades, poco más de la mitad de los padres, el 51,6\% consideran que, después de hacer los deberes, a los niños les queda poco tiempo disponible para hacer otras cosas. Cuando se pregunta a los padres si estiman que la cantidad de tareas perjudica las actividades en familia, el 34,4\% responde 
afirmativamente y el 65,6\% entiende que existe ese perjuicio.

\section{Verificación o corrección de los TPC}

La verificación y corrección constituyen la etapa final en el ciclo de los TPC. Este es un momento clave en su desarrollo metodológico, puesto que proporciona a los alumnos el feedback necesario para identificar cuáles son sus logros y cuáles sus áreas de mejora. Esta información es fundamental para que el alumno construya una imagen correcta de sí mismo como aprendiz y constituye la base para la autorregulación de sus futuros aprendizajes. Además, la corrección de los TPC aporta al profesor una información muy valiosa acerca del desarrollo del proceso de enseñanza- aprendizaje. En este sentido, los resultados del estudio corroboran que los alumnos son conscientes de la importancia de la corrección, ya que el 93,8\% estima que la corrección de los TPC es importante para ellos.

Sin embargo, los datos de este estudio muestran que los profesores no siempre corrigen los TPC. Según el 41,3\% de los alumnos, los profesores corrigen a veces las tareas y $1,3 \%$ refiere que nunca son corregidas. Como contrapartida el 57,5\% declara que los profesores corrigen siempre los trabajos que hacen en casa. Por su parte, el $75 \%$ de los profesores afirma corregir siempre los TPC, mientras que el $25 \%$ reconoce que los corrige a veces. Preguntados por la forma de corrección, un 62,5\% de los alumnos refiere que el profesor recoge los trabajos y los devuelve corregidos, el 35\% declara que la corrección se realiza colectivamente en la clase y el 2,5\% explica que la corrección se realiza oralmente. Las respuestas de los profesores difieren en los porcentajes con las de los alumnos, puesto que la mitad expone que la corrección se realiza conjuntamente en la clase, el 25\% explica que recoge los TPC y los devuelve corregidos y el 25\% restante refiere corregir los TPC individualmente con los alumnos. En cuanto a los instrumentos de evaluación de los TPC, el 75\% afirma poseer y utilizar un instrumento de evaluación y el 25\% reconoce no contar con ninguno.

En definitiva, las prácticas detectadas en el proceso de corrección de los TPC indican que los profesores no aprovechan todo el potencial del feedback para contribuir de manera efectiva al proceso de aprendizaje. El $25 \%$ del profesorado reconoce que a veces no corrige los TPC y según el 41,3\% de los alumnos los TPC no son siempre corregidos. La corrección conjunta en clase de los TPC, referida por el 50\% del profesorado como práctica habitual, no es el instrumento más adecuado para que alumnos y profesores obtengan información sobre los logros y áreas de mejora. La ausencia de corrección sistemática de los TPC se ha detectado en estudios anteriores. Simões (2012, p. 30) revela que el $65 \%$ de los alumnos afirman que los profesores corrigen "casi siempre" sus TPC y Henriques (2006, p. 237) recoge testimonios que evidencian que no siempre se corrigen las tareas, por lo que concluye que la fase de corrección de los TPC “revela alguns indicadores significativos de uma prática que sofre de negligência.

Por otro lado, durante la observación en el aula se han constatado algunas derivas negativas en la verificación y corrección de los TPC. Al comprobar la realización de los TPC, los profesores preguntan a los alumnos las causas por las que, en su caso, no se llevaron a cabo. Estos momentos se traducen en una situación de tensión en el aula y los alumnos declaran que cuando no hacen los deberes, suelen copiarlos de sus compañeros, mentir al profesor sobre la causa del incumplimiento e incluso faltar a clase. Estos constituyen 
efectos indeseados que desembocan en una perversión del sistema, puesto que de nada sirve que los profesores encarguen una buena cantidad de TPC si luego los alumnos los van a copiar o van a hacerlos de cualquier manera para salir del paso y no reflexionan ni se implican cognitivamente en la tarea.

\section{Conclusiones y recomendaciones}

En esta investigación se han estudiado las perspectivas de profesores, padres y alumnos de $2^{\circ}, 3^{\circ}$ y $4^{\circ}$ de educación primaria de un colegio público situado en Lisboa respecto de las tres etapas que conforman la práctica educativa de los TPC: prescripción, realización y corrección. Los resultados obtenidos conforman que la mayoría de los profesores prescriben diariamente TPC, especialmente en $3^{\circ}$ año. El tiempo de realización media de los deberes es inferior a una hora, aunque existen discordancias a este respecto entre los diversos actores, de manera que los profesores estiman que los alumnos necesitan menos tiempo del que estos declaran emplear y los padres consideran que les lleva más tiempo. Tras hacer los deberes, la mayoría de los padres $(51,6 \%)$ opina que queda poco tiempo para hacer otras actividades y algo más de un tercio considera que las tareas perjudican las actividades en familia. No obstante, la mayoría de los padres está de acuerdo con la prescripción de los TPC y considera que contribuyen a la adquisición de los aprendizajes. En cuanto al tipo de TPC, los más frecuentes son ejercicios del libro y cuentas y un 50\% del profesorado relata prescribir tareas diferenciadas. La mayoría de los alumnos reconoce necesitar ayuda para realizar sus TPC y un alto porcentaje de padres refiere prestar auxilio a sus hijos en las tareas, a pesar de que los profesores están divididos en lo que respecta a los beneficios de la implicación parental, puesto que un 25\% opina que los padres no deberían ayudar, un $50 \%$ solo a veces y el $25 \%$ se posiciona a favor de la participación familiar en los TPC. En cuanto a la corrección, se ha detectado que no siempre se lleva a cabo y la práctica mayoritaria es la corrección en gran grupo.

Los resultados obtenidos revelan unas prácticas tradicionales de prescripción de TPC que ya habían sido detectadas en estudios anteriores (HENRIQUES, 2006; SIMÃO, 2012), y que han llevado a sugerir la necesidad de “cambios urgentes” (SIMÃO, 2012, p. 17). Los principales aspectos que precisan de reflexión son la frecuencia y tipo de tareas, la participación parental y la corrección y evaluación.

La frecuencia diaria de las tareas limita el desarrollo de la autonomía y autorregulación de los alumnos. Una organización semanal de las tareas daría un mayor margen al alumno para intervenir más activamente en la gestión de su tiempo y favorecería una organización más flexible a nivel personal y familiar. Por otro lado, la investigación insiste en que para que los TPC sean eficaces, han de ser significativos y centrarse en la resolución de problemas del mundo real (REBELO; CORREIA, 1999; SHERNOFF; CSIKSZENTMIHALYI; SCHNEIDER; SHERNOFF, 2003). Hacer fichas del libro, cuentas o copias, que son las tareas más frecuentes a tenor de este estudio, no cumplen con este objetivo. Es conveniente que la prescripción de TPC se inspire en los principios formulados por Alleman et al., (2010): que aporten una aplicación práctica en el mundo real de los saberes adquiridos en la escuela, que construyan el conocimiento y potencien el pensamiento crítico, que lleven la educación al hogar y a la comunidad implicando a los adultos a través de una cooperación fructífera entre familia y escuela; que aprovechen la diversidad de 
los estudiantes como fuente de aprendizaje, que se empleen para personalizar y mantener actualizado el currículo y que proporcionen oportunidades de aprendizaje a través de la exploración del entorno.

Por otro lado, la participación parental constituye uno de los elementos más importantes para que la práctica de los TPC se convierta en un instrumento eficaz. Sin embargo, como hemos visto, no toda la implicación de los padres es positiva, por lo que sería interesante que los profesores contaran con la formación necesaria para asesorar a los padres y establecer espacios de colaboración y coordinación entre familias y escuela (VILLAS-BONAS, 2000). Walker, Hoover-Dempsey, Whetsel, y Green (2004) identifican, entre las formas de intervención parental eficaz, las siguientes: Interacción con los profesores respecto los TPC (valorar el colegio y el aprendizaje, hablar con el tutor, responder a las demandas educativas del colegio); organización del ambiente físico y psicológico para facilitar la realización de los TPC (ayudar al alumno a estructurar el tiempo, espacio físico y materiales; asegurar la presencia, disponibilidad y ayuda parental), proporcionar supervisión en el proceso de realización de los TPC (supervisión y monitorización de los TPC, atender a las señales de éxito o dificultad del alumno; motivarlo), respuesta a los resultados del desempeño de los TPC (reconocer y ofrecer apoyo emocional, incentivar la competencia y esfuerzo; revisar, verificar y corregir los TPC).

La corrección y la evaluación son claves para que el alumno pueda aprender a través de la realización de los TPC (MEIRIEU, 1998). Para Rebelo y Correia (1999), los TPC son eficaces cuando el profesor los recoge y los devuelve corregidos con comentarios, mientras que Stoeger e Ziegler (2011) proponen una intervención basada en la monitorización y autorregulación. Según estos autores, los TPC despliegan todos sus beneficios cuando se ejercita a los alumnos en estrategias de autorregulación de sus aprendizajes, no solo en lo que se refiere a la gestión del tiempo sino también a la detección de los propios puntos fuertes y las áreas de mejora, es decir, cuando los alumnos autoevalúan el proceso y el resultado. La autoevaluación, las discusiones en grupo y del modelaje de hábitos de trabajo y resolución de tareas contribuye al desarrollo de la autonomía y la autorregulación y por ende, a la mejora del proceso de aprendizaje.

En definitiva, los datos muestran que no se está aprovechando todo el potencial de los TPC y que es necesario introducir metodologías más eficaces que conviertan a esta práctica en una herramienta realmente valiosa para mejorar el aprendizaje y el rendimiento escolar.

\section{Referências}

ALLEMAN, J.; BROPHY, J.; KNIGHTON, B.; LEY, R.; BOTWINSKI, B.; MIDDLESTEAD, S. Homework done right: powerful learning in real-life situations. Thousand Oaks, CA: Corwin Press, 2010.

ANTUNES, C. S. Os trabalhos para casa numa escola de $10^{\circ}$ ciclo do ensino básico. 2012. 93 f. Dissertação (Mestrado em Educação) - Escola Superior de Educação, Instituto politécnico de Castelo Branco, Castelo Branco, 2012.

ARAÚJO, M. J. “Trabalhos de casa” uma questão na ordem do dia. Observatório, n. 185, p. 90-91, 2009.

BALLI, S. J. When mom and dad help: student reflections on parent involvement with homework. Journal of Research and 
Development in Education, n. 31(3), p. 142-148, 1998.

BEMBENNUTTY, H. Self-regulation of homework completion. Psychology Journal, n. 6, p. 138-153, 2009.

Meaningful and maladaptive homework practices: The role of selfefficacy and self-regulation. Journal of Advanced Academics, n. 22(3), p. 448-473, 2011.

BEMPECHAT, J. The motivational benefits of homework: a social-cognitive perspective. Theory in Practice, 43, p. 189-196, 2004.

CARVALHO, M. E. P; SERPA, M. H. B. Dever de casa: visões de mães e professoras. Olhar de professor, Ponta Grossa, n.9 (1), p. 31-46, 2006.

COOPER, H. Homework. White Plains, NY: Longman, 1989.

Homework for all - in moderation.

Educational Leadership, n. 58, p. 34- 38, 2001.

COOPER, H.; LINDSAY, J.J.; NYE, B. Homework in the home: How student, family and parenting style differences relate to the homework process. Contemporary Educational Psychology, n. 25 p. 464-487, 2000.

COOPER, H.; LINDSAY, J. L.; NYE, B.; GREATHOUSE, S. Relationships among attitudes about homework, amount of homework assigned and completed, and student achievement. Journal of Educational Psychology, n. 90, p. 70-83, 1998. Doi: 10.1037/0022-0663.90.1.70.

COOPER, H.; ROBINSON, J. C.; PATALL, E. A. Does homework improve academic achievement?: A synthesis of research. Review of Educational
Research, n. 76, p. 1-62, 2006. Doi: 10.3102/00346543076001001

COOPER, H.; VALENTINE, J. C. Using research to answer practical questions about homework. Educational Psychologist, n. 36, p. 143-153, 2001.

CORNO, L.; XU, J. Homework as the job of childhood. Theory into Practice, n. 43(3), p. 227-233, 2004.

DENZIN, N. ET AL. O planeamento da pesquisa qualitativa: teoria e abordagens. Porto Alegre: Ed. Artmed, 2006.

DUDLEY-MARLING, C. How school troubles come home: the impact of homework on families of struggling learners. Current Issues in Education, n. 6, 2003. Disponível em: <http://cie.ed.asu.edu/ volume6/number4>

EPSTEIN, J. L. Homework practices, achievements and behaviors of elementary school students (Report No. 27). Baltimore, MD: Johns Hopkins University, Center for Research on Elementary and Middle Schools, 1988.

GALLOWAY, M.; CONNER, J.; POPE, D. Nonacademic Effects of Homework in Privileged, High-Performing High Schools, The Journal of Experimental Education, n. 81:4, p. 490-510, 2013.

GONZÁLEZ-PIENDA, J. A.; NÚÑEZ, J. C. La implicación de los padres y su incidencia en el rendimiento de los hijos. Revista de Psicología y Educación, n. 1(1), p. 115-134, 2005.

HENRIQUES, M. E. Os trabalhos de casa na escola do $1 .^{\circ}$ ciclo da Luz: estudo de caso. Interacções, n. 2, p. 220-243, 2006.

HONG, E.; WAN, M.; PENG, Y. Discrepancies between students and teachers perceptions of homework. Journal of 
Advances Academics, n. 22(2), p. 280-308, 2011.

KIEWRA, K. A.; KAUFFMAN, D. F.; HART, K.; SCOULAR, J.; BROWN, M.; TYLER, B. What parents, researchers and the popular press have to say about homework, scholarlypartnershipsedu, n. 4(1), Article 7, 2009. Disponível em: <http://opus.ipfw. edu/spe/vol4/iss1/7>

KITSANTAS, A.; ZIMMERMAN, B.J. College students' homework and academic achievement: the mediating role of selfregulatory beliefs. Metacognition and Learning, n. 4, p. 97-110, 2009.

KOHN, A. The homework myth: why our kids get too much of a bad thing. Cambridge, MA: Da Capo Press, 2006.

KRALOVEC, E.; BUELL, J. The end of homework: how homework disrupts families, overburdens children and limits learning. Boston, MA: Beacon Press Books, 2000.

LEVIN, I.; LEVY-SHIFFF, R.; APPELBAUM-PELED, T.; KATZ, I.; KOMAR, M.; MEIRAN, N. Antecedents and consequences of maternal involvement in children's homework: a longitudinal analysis. Journal of Applied Developmental Psychology, n. 18 (2), p. 207-227, 1997.

MEIRIEU, P. Os trabalhos de casa. Lisboa: Editorial Presença, 1998.

MARZANO, R. J.; PICKERING, D. J. The case for and against homework. Educational Leadership, n. 64(6), p. 74-79, 2007.

PAN, I.; REGUEIRO, B.; PONTE, B.; RODRÍGUEZ, S.; PIÑEIRO, I.; VALLE, A. Motivación, implicación en los deberes escolares y rendimiento académico. Aula Abierta, n. 41(3), p. 13-22, 2013.
PATALL, E. A.; COOPER, H.; ROBINSON, J. C. (2008). Parent Involvement in Homework: A Research Synthesis. Review of Educational Research, n. 78(4), p. 10391101, 2008.

PIRES, S. I. L. Os trabalhos para casa no $1^{\circ}$ Ciclo do Ensino básico: a visão das crianças e dos pais. 2012. 128 f. Dissertação (Mestrado em Educação) - Escola Superior de Educação, Instituto politécnico de Castelo Branco, Castelo Branco, 2012.

REBELO, J.; CORREIA, O. O sentido dos deveres para casa. Coimbra: Gráfica de Coimbra, 1999.

ROSÁRIO, P.; MOURÃO, R.; NÚÑEZ, J. C.; GONZÁLEZ-PIENDA, J.; VALLE, A. SRL and EFL homework: gender and grade effects. Academic Exchange Quarterly, n. 10(4), p. 135-140, 2006.

SHERNOFF,D.J.;CSIKSZENTMAIHALYI, M.; SCHNEIDER, B.; SHERNOFF, E. S. Student engagement in high school classrooms from the perspective of flow theory. School Psychology Quarterly, n. 18, p. 158-176, 2003.

SIMÃO, A. M., DUARTE, F., FERREIRA, P. C. Trabalhos para casa como ferramento autorregulatória: perspectivas e implicações para as práticas educativas. Cuadernos de Educação, n.42, p. 12-44, 2012.

SOUSA, M. J.; BAPTISTA, C. S. Como fazer investigação, teses, dissertações e relatórios. Lisboa: LIDEL, 2011.

SHERNOFF,D.J.;CSIKSZENTMAIHALYI, M.; SCHNEIDER, B.; SHERNOFF, E. S. Student engagement in high school classrooms from the perspective of flow theory. School Psychology Quarterly, n. 18, p. 158-176, 2003.

STOEGER, H; ZIEGLER, A. Selfregulatory training through elementary - 
school students' homework completion. In: SCHUNK, D.; ZIMMERMAN, B. (Ed.). Handbook of Self- Regulated Learning and performance. Routledge, New York, 2011. p. 1-12.

TRAUTWEIN, U.; KOLLER, O. The relationship between homework and achievement - still much of a mystery. Educational Psychology Review, n. 15 (2), p. 115-145, 2003.

VILLAS-BOAS, M. A parceria entre a escola, a família e a comunidade: trabalho de casa pra o desenvolvimento da literacia. Lisboa: Ministério da Educação, 2000.

WALKER, J.; HOOVER-DEMPSEY, K. V.; WHETSEL, D. R.; GREEN, C. Parental involvement in homework: a review of current research and its implications for teachers, after school program staff, and parent leaders. Cambridge, MA: Harvard Family Research Project, 2004.

ZABALZA, M, A. Planificação e desenvolvimento curricular na escola. Lisboa: Edições Asa, 2001.

\section{ANEXO 1. QUESTIONARIO PARA ALUMNOS}

Responde às questões seguintes, colocando um $x$ na opção que consideras ser mais correta.

1. Quantas vezes, por semana, tens TPC?

$\square$ Só aos fins de semana

$\square 1$ a 2 vezes

$\square 3$ a 4 vezes

$\square$ Todos os dias

2. Quanto tempo, aproximadamente, demoras a fazer os teus TPC?

$\square$ Menos de $15 \mathrm{~min}$

$\square$ Entre 15 a $30 \mathrm{~min}$.

$\square$ Entre 30 a $45 \mathrm{~min}$.

$\square$ Entre $45 \mathrm{~min}$. a $1 \mathrm{~h}$

$\square$ Mais de $1 \mathrm{~h}$.

3. Indica 2 tipos de TPC que levas com maior frequência? 
$\square$ Cópias

Cálculos

Leitura

Fichas do livro

Fichas elaboradas pelo professor

$\square$ Pesquisas

Outros. Quais?

4. Quando tens TPC, sabes como realizá-lo?

$\square \operatorname{Sim}$

$\square$ Às vezes

$\square$ Não

5. Quando não fazes os TPC é devido:

$\square$ À falta de tempo

$\square$ À disciplina

$\square$ Ao tipo de trabalho (estudar, resolver exercícios de matemática, interpretação de textos...)

$\square$ À Falta de materiais (computador, dicionário...)

6. Onde costumas realizar os TPC?

$\square \mathrm{Na}$ escola

$\square$ Em casa

$\square \mathrm{NaCAF}$

$\square$ No explicador 


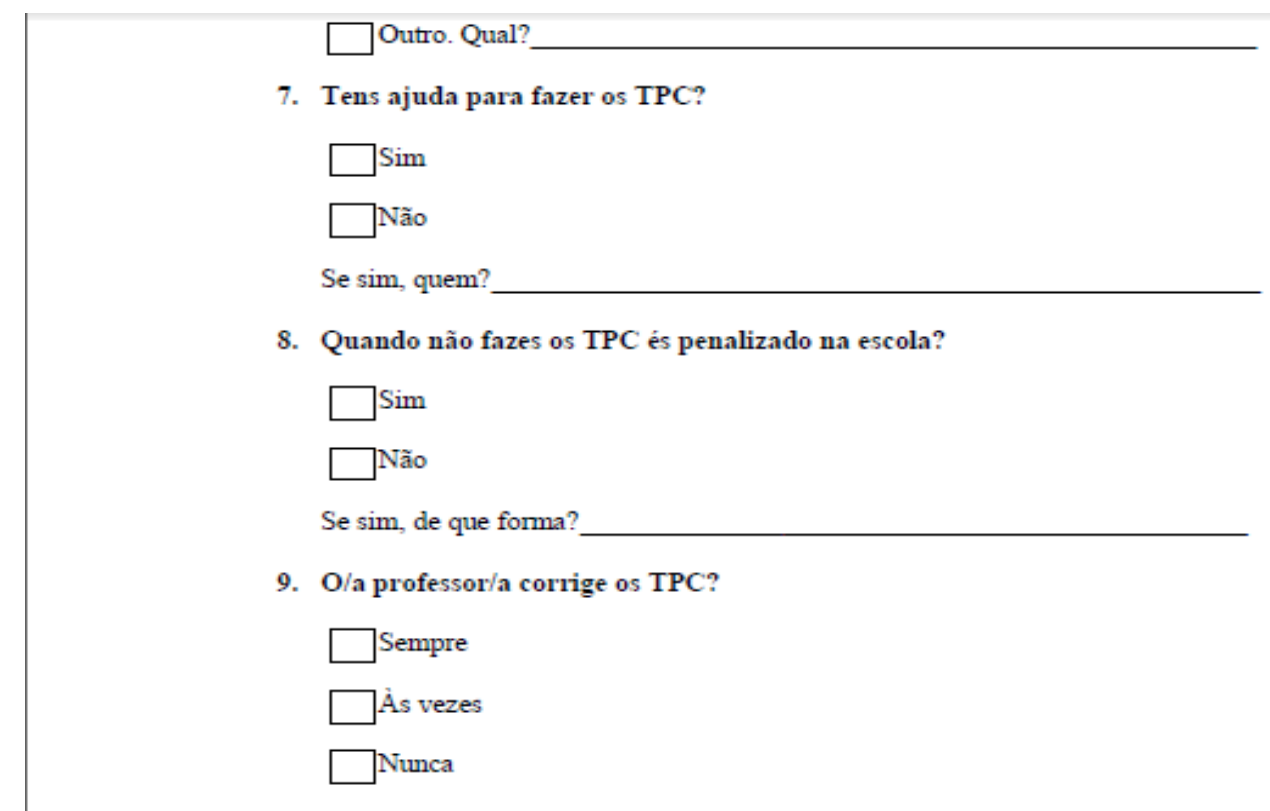

10. Se corrige os TPC como é o que o faz?

$\square$ No quadro

$\square$ Oralmente

$\square$ Recolhe e devolve os trabalhos corrigidos

$\square$ Individualmente com os alumos

11. A correção do TPC para ti é:

$\square$ Muito importante

$\square$ Importante

$\square$ Nada importante

12. Na tua opinião, a realização de TPC é importante? Porquê?

Obrigada pela tua colaboração 